\title{
Gadolinium Doped Layered Double Hydroxides for Simultaneous Drug Delivery and Magnetic Resonance Imaging
}

\author{
Ziwei Zhang ${ }^{1,2} \cdot$ Connor J. R. Wells ${ }^{2} \cdot$ Ruizheng Liang $^{3} \cdot$ Gemma-Louise Davies $^{2} \cdot$ Gareth R. Williams $^{1}$
}

Received: 8 December 2021 / Accepted: 11 January 2022 / Published online: 3 February 2022

(C) The Author(s) 2022

\begin{abstract}
In this study, gadolinium (Gd) doped MgAl layered double hydroxides (LDHs) were synthesized via a 'bottom-up' method and fully characterized by X-ray diffraction, infrared spectroscopy and relaxivity measurements. Two cytotoxic agents were then intercalated via ion-exchange. X-ray diffraction patterns exhibit expanded interlayer spacings as a result of successful drug intercalation. Infrared spectra also showed characteristic peaks of the incorporated methotrexate (MTX) or 5-fluorouracil (5-FU). The LDHs were found to be highly stable under physiological conditions, while in acidic conditions a small proportion of Gd was freed into the immersion medium. Dissolution tests revealed that both 5FU and MTX were rapidly released from the LDH carrier. The longitudinal relaxivity of Gd-LDHs remains largely stable during drug release over $24 \mathrm{~h}$, and was higher in acidic environments. Overall, the drug-loaded Gd-LDH systems prepared in this study could serve as $\mathrm{pH}$-sensitive theranostic platforms for MRI-guided anti-cancer therapy.
\end{abstract}

Keywords Layered double hydroxide $\cdot$ Magnetic resonance imaging $\cdot$ Drug delivery $\cdot$ Theranostics

\section{Introduction}

Layered double hydroxide (LDH) nanocomposites have been widely explored in biomedical applications because of their biocompatibility, low toxicity and potential for cellular permeability [1]. For example, hydrotalcite, a classic MgAl-LDH with the formula $\left[\mathrm{Mg}_{6} \mathrm{Al}_{2}\left(\mathrm{CO}_{3}\right)(-\right.$ $\mathrm{OH})_{16} \cdot 4 \mathrm{H}_{2} \mathrm{O}$ ] has been commercialized by Bayer, with the product name of "Talcid®" [2]. It is prescribed for neutralizing the gastric acid of patients with acute or chronic gastritis.

Gemma-Louise Davies

gemma-louise.davies@ucl.ac.uk

$\bowtie$ Gareth R. Williams

g.williams@ucl.ac.uk

1 UCL School of Pharmacy, University College London, 29 39 Brunswick Square, London WC1N 1AX, UK

2 UCL Department of Chemistry, University College London, 20 Gordon St, London WC1H 0AJ, UK

3 State Key Laboratory of Chemical Resource Engineering, Advanced Innovation Center for Soft Matter Science and Engineering, Beijing University of Chemical Technology, Beijing 100029, China
A key advantage of LDH-based drug-delivery systems is that active agents intercalated can be released in a controlled manner at the target area. This capacity for localised release can protect the agents from degradation and alleviate undesirable release outside the target site. This can minimise side effects, avoid toxicity and reduce dosing concentration and frequency, thereby improving the overall bioactivity [3, 4]. For instance, podophyllotoxin, a highly cytotoxic chemotherapeutic drug with poor water solubility and low bioavailability, has been intercalated into an LDH vehicle. Compared to the pure drug, in vitro cell experiments proved that the LDH nanocomposite enhanced the cytotoxicity, suggesting that LDHs can promote cell penetration, increase the stability of the active agent, and release a drug payload locally in a controlled manner [5].

Another characteristic of LDH nanocomposite delivery systems is their $\mathrm{pH}$ sensitive properties. This is related to the nature of metal hydroxides. A number of experiments have revealed that in acidic conditions (e.g. $\mathrm{pH} 4.8$, which mimics the environment in lysosomal conditions), the release of a drug from LDH nanocomposites is faster than at the physiological pH (7.4) [3, 6]. According to Rives et al. drug release from the interlayer area is mainly dependent on two mechanisms: ion-exchange or weathering (dissolution of the layers) [7, 8]. At the physiological 
$\mathrm{pH}$, it is driven by ion-exchange, while at acidic $\mathrm{pH}$ both ion-exchange and weathering are in play [9].

Nanomaterials are promising vehicles for use in theranostics because of their capacity to integrate different agents in a well-organized manner, and their potential to target a specific area of disease. Among the wide variety of possible bioimaging techniques, magnetic resonance imaging (MRI) stands out because of its ability to provide high spatial and temporal resolution without the use of ionizing radiation. To ensure high-quality images, exogenous contrast agents (CAs) are typically employed to enhance the MRI signal. Small molecule CAs have paved the way for improved MRI imaging and facilitated medical diagnosis. However, they have a number of drawbacks, such as rapid elimination, which has led to the development of higher molecular weight species [10]. Macromolecular MRI CAs have attracted attention because of their high contrast efficiency and versatile dignostic and therapeutic functionality. In addition, a number of $\mathrm{Gd}$ based nanoparticles can provide high MRI contrast, for instance gadolinium-based metal-organic frameworks [11, 12], gadofullerenes [13], poly(acrylic acid) coated gadolinium fluoride [14], $\mathrm{GdBO}_{3}-\mathrm{Fe}_{3} \mathrm{O}_{4}$ [15], organic acid functionalised $\mathrm{Gd}_{2} \mathrm{O}_{3}$ [16], $\mathrm{NaGdF}_{4}$ [17], or layered gadolinium hydroxides [18]. However, these often display low kinetic stability and thus potentially have high risks of toxicity.

LDHs can be easily doped with a small amount of paramagnetic metal ions to obtain high MRI relaxivity [19-21]. Further, the coordinating hydroxide groups can be protonated, providing a $\mathrm{pH}$-responsive property which can be utilized in cancer diagnosis and treatment. A number of Gd-doped LDHs have been developed as multifunctional platforms for both bioimaging and treatment [22-24]. Other ions have also been explored. For example, a manganese doped LDH was developed as a $\mathrm{T}_{1}$-MRI contrast agent sensitive to $\mathrm{pH}$ changes in the physiological range [25]. In this work, we synthesized a $\mathrm{Gd}^{3+}$ doped $\mathrm{LDH}$ using a 'bottom-up' approach, and used this to intercalate cytotoxic drugs (5-fluorouracil and methotrexate), as depicted in Fig. 1. The resultant materials were fully characterized and their in vitro drug release and relaxivity profiles were explored in detail.

\section{Methods}

\section{Materials}

Chemicals were sourced as follows: sodium hydroxide and anhydrous ethanol were purchased from Fisher Scientific Ltd; sodium chloride, xanthan gum, $\mathrm{AlCl}_{3} \cdot 6 \mathrm{H}_{2} \mathrm{O}, \mathrm{MgCl}_{2}$ $4 \mathrm{H}_{2} \mathrm{O}, \mathrm{GdCl}_{3} \cdot 6 \mathrm{H}_{2} \mathrm{O}$ and fluorouracil $(5 \mathrm{FU})$ were obtained from Sigma-Aldrich. Methotrexate (MTX) was purchased from Aladdin Co., Ltd. Ultrapure water was collected from a Millipore MilliQ system operated at 18.2 M .

\section{Preparation of Layered Double Hydroxides (MgAl-LDH)}

The reaction was based on a previous report in the literature [17]. In a typical procedure, an aqueous solution $(10 \mathrm{~mL})$ containing $\mathrm{MgCl}_{2} \cdot 4 \mathrm{H}_{2} \mathrm{O}\left(0.5 \mathrm{M}, 167.3 \mathrm{~g} \mathrm{~mol}^{-1}\right.$, $0.8364 \mathrm{~g})$ and $\mathrm{AlCl}_{3} \cdot 6 \mathrm{H}_{2} \mathrm{O}\left(0.25 \mathrm{M}, 241.4 \mathrm{~g} \mathrm{~mol}^{-1}\right.$, $0.6036 \mathrm{~g}$ ) was quickly added into aqueous $\mathrm{NaOH}$ solution (40 mL, $0.4 \mathrm{M}, 40 \mathrm{~g} \mathrm{~mol}^{-1}, 0.64 \mathrm{~g}$ ) while stirring vigorously. After stirring for $20 \mathrm{~min}$, the $\mathrm{Mg}_{2} \mathrm{Al}-\mathrm{LDH}$ slurry was collected via centrifugation (9000 rpm, $20 \mathrm{~min}$ ), washed twice with $20 \mathrm{~mL}$ of deionized water, and re-suspended in deionized water $(40 \mathrm{~mL})$. Then, the white slurry was magnetically stirred $(100 \mathrm{rpm})$ and heated at $100{ }^{\circ} \mathrm{C}$ in a $100 \mathrm{~mL}$ round-bottomed flask for $6 \mathrm{~h}$. Finally, the $\mathrm{LDH}$ slurry was centrifuged (9000 rpm, $20 \mathrm{~min}$ ), washed with deionized water three times, and a transparent and homogeneous suspension obtained after re-dispersion in $10 \mathrm{~mL}$ of deionized water.

\section{Synthesis of Gd-Doped LDHs (Gd-LDH)}

$10 \mathrm{~mL}$ of an aqueous salt solution containing $\mathrm{MgCl}_{2} \cdot 4 \mathrm{H}_{2} \mathrm{O}$ $\left(0.5 \mathrm{M}, 167.3 \mathrm{~g} \mathrm{~mol}^{-1}, 0.8364 \mathrm{~g}\right), \mathrm{AlCl}_{3} \cdot 6 \mathrm{H}_{2} \mathrm{O}(0.237 \mathrm{M}$, $\left.241.4 \mathrm{~g} \mathrm{~mol}^{-1}, 0.5122 \mathrm{~g}\right)$, and $\mathrm{GdCl}_{3}(0.013 \mathrm{M}, 371.7 \mathrm{~g}$ $\mathrm{mol}^{-1}, 0.0483 \mathrm{~g}$ ) was quickly added into an aqueous $\mathrm{NaOH}$ solution $\left(40 \mathrm{~mL}, 0.4 \mathrm{M}, 40 \mathrm{~g} \mathrm{~mol}^{-1}, 0.64 \mathrm{~g}\right.$ ) while stirring vigorously. After stirring for $20 \mathrm{~min}$, the Gd-LDH slurry was magnetically stirred $(100 \mathrm{rpm})$ and heated at $110{ }^{\circ} \mathrm{C}$ for $4 \mathrm{~h}$ in a $100 \mathrm{~mL}$ round bottomed flask. Finally, the Gd-LDH slurry was centrifuged (9000 rpm, $20 \mathrm{~min}$ ), washed with deionized water three times, and a transparent and homogeneous suspension obtained after re-dispersion in $10 \mathrm{~mL}$ of deionized water.

\section{Drug Loading of Gd-Doped LDHs}

$400 \mathrm{mg}$ of Gd-LDH was suspended in $20 \mathrm{~mL}$ of water and $390 \mathrm{mg}$ of $5 \mathrm{FU}$ or $455 \mathrm{mg}$ of MTX was added to the solutions, keeping the $\mathrm{pH}$ at 9.5 . After shaking at $40{ }^{\circ} \mathrm{C}$ for $48 \mathrm{~h}$ (100 rpm), 5FU or MTX-loaded Gd-LDHs (5FU-GdLDH or MTX-Gd-LDH) were collected after washing with water: ethanol $(1: 1 \mathrm{v} / \mathrm{v})$ three times.

\section{Characterization}

A field emission scanning electron microscope (SEM; FEI Quanta 200F) connected to a secondary electron detector (Everheart-Thornley Detector-ETD) was employed to 
Fig. 1 Schematic illustration of the production of drug loaded Gd-LDH particles

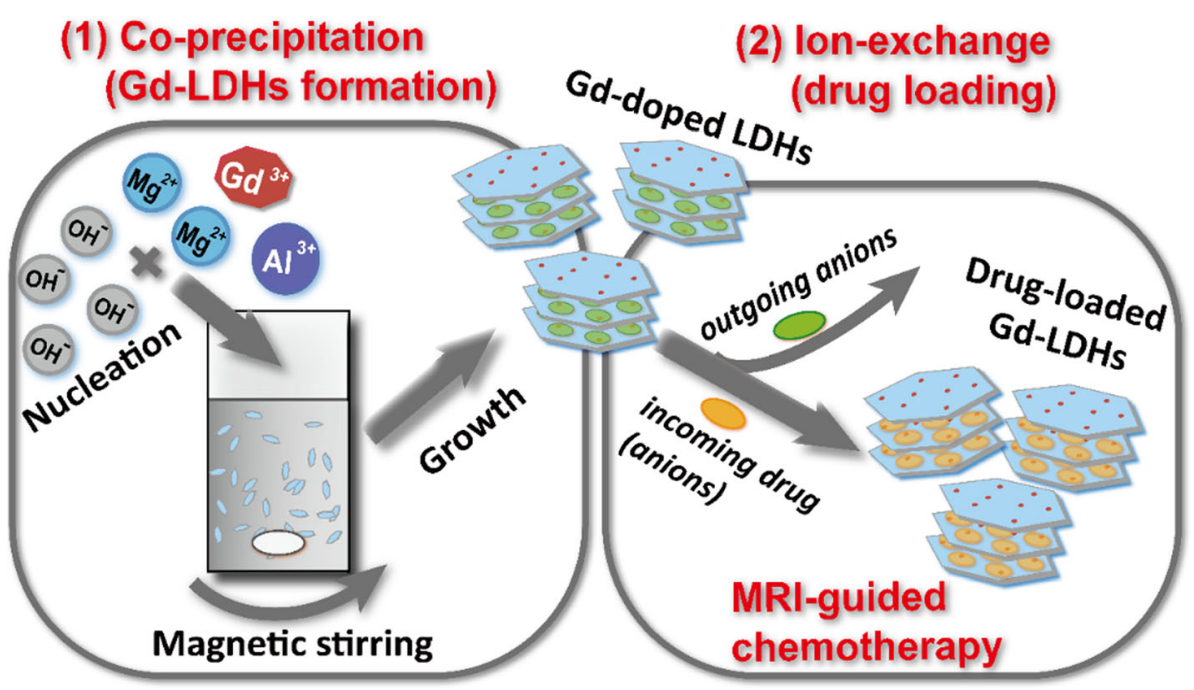

image the LDH materials. Samples were coated with a $20 \mathrm{~nm}$ gold-sputter (Quorum Q150T) prior to imaging. Transmission electron microscope (TEM) images were obtained on a JEOL JEM-1200 microscope operated at $120 \mathrm{kV}$ with a beam current of ca. $80 \mathrm{~mA}$. A Gatan Orius 11 megapixel camera was used to take images. The size distribution was determined from the TEM micrographs by using the ImageJ software (version $1.52 \mathrm{~s}$, National Institutes of Health) to measure the diameter of at least 100 particles. Fourier transform infrared (FTIR) analyses were carried out on a Spectrum 100 instrument (PerkinElmer) over the range $650-4000 \mathrm{~cm}^{-1}$ at a resolution of $4 \mathrm{~cm}^{-1}$. A MiniFlex 600 diffractometer (Rigaku) supplied with $\mathrm{Cu}$ $\mathrm{K} \alpha$ radiation was used to collect $\mathrm{X}$-ray diffraction (XRD) patterns $(\lambda=0.15418 \mathrm{~nm}, 40 \mathrm{kV}, 15 \mathrm{~mA})$. Patterns were recorded over the $2 \theta$ range from $3^{\circ}$ to $70^{\circ}$ (step $=0.01^{\circ}$ ). Energy dispersive X-ray spectroscopy (EDX) was carried out using a Carl Zeiss EVO 25 SEM with an Oxford Instruments X-max 80 EDS detector. The accelerating voltage was $20 \mathrm{kV}$, with a working distance of $8.5 \mathrm{~mm}$. The Oxford Instruments Aztec 5.0 software was used to collect EDX data.

The hydrodynamic diameters of the LDHs were measured on a Zetasizer Nano ZS instrument (Malvern Instruments). $2 \mathrm{mg}$ of sample was dispersed into $2 \mathrm{~mL}$ of deionized water and sonicated for $30 \mathrm{~min}$ before measurements. A $4 \mathrm{~mW} \mathrm{He-Ne} 633 \mathrm{~nm}$ laser module was employed and scattered light was measured at $173^{\circ}$ (back scattering). The attenuator and position were selected automatically by the instrument and particle sizes are reported as the average of three independent measurements. Zeta potential measurements were performed at
$25{ }^{\circ} \mathrm{C}$. Samples were loaded into a DTS1070 disposable folded capillary polystyrene cell (Malvern Instruments). The zeta potential was calculated with the Zetasizer software version 7.11 (Malvern Instruments), using Smoluchowski's equation with a Henry's function $[\mathrm{f}(\mathrm{K} \alpha)]$ of 1.50. X-ray photoelectron spectroscopy (XPS) measurements were carried out on an Axis Supra+ system (Kratos, Japan) with an $\mathrm{Al} \mathrm{K} \alpha \mathrm{X}$-ray source $(15 \mathrm{kV}, 10 \mathrm{~mA})$. The C1s peak was corrected to $284.8 \mathrm{eV}$ for energy calibration. [Gd] (mM) was quantified on a Varian 720-ES inductively coupled plasma optical emission spectrometer (ICP-OES). Samples were digested using a few drops of concentrated $\mathrm{HNO}_{3}$, and then diluted to $10 \mathrm{~mL}$ with DI water.

\section{Stability Test}

Composite stability was explored in PBS buffer $(\mathrm{pH} 6.5$ or 7.4) and acetate buffer ( $\mathrm{pH} 5.0) .50 \mathrm{mg}$ of sample $(\mathrm{n}=3)$ was dispersed in $5 \mathrm{~mL}$ of the relevant buffer and transferred to a dialysis bag $(\mathrm{MWCO}=3500 \mathrm{Da})$. The bag was submerged into $15 \mathrm{~mL}$ of the same buffer and stirred at $37{ }^{\circ} \mathrm{C}(50 \mathrm{rpm}) .1 \mathrm{~mL}$ aliquots were withdrawn from the medium at predetermined times and analysed by ICP-OES on an Agilent 7500cx spectrometer to determine the free [Gd]. To maintain a constant volume, $1 \mathrm{~mL}$ of fresh preheated buffer was added to the dissolution vessel at each time point.

\section{In Vitro Drug Release}

Loading capacity (LC \%) and encapsulation efficiency (EE $\%$ ) were measured using UV spectroscopy at $262 \mathrm{~nm}$ for 
5FU and $303 \mathrm{~nm}$ for MTX (Cary 100 instrument, Agilent). $2 \mathrm{mg}$ of drug loaded Gd-doped LDH powder was charged in a $10 \mathrm{~mL}$ volumetric flask and suspended in $5 \mathrm{~mL}$ of DI water. The solution was acidified with one droplet of concentrated $\mathrm{HCl}$, and shaken until the $\mathrm{LDH}$ was fully dissolved. After neutralizing with $0.2 \mathrm{M}$ aqueous $\mathrm{NaOH}$, the solutions were made up to $10 \mathrm{~mL}$ with deionized water. The resultant solution was filtered through a $0.22 \mu \mathrm{m}$ PVDF-type syringe filter before being analysed with UV spectroscopy.

Release experiments were undertaken on a magnetic stirrer (C-MAG HS 7, IKA) with temperature control. $20 \mathrm{mg}$ samples $(\mathrm{n}=3)$ were dispersed in $5 \mathrm{~mL}$ of a buffer (pH 7.4 PBS buffer, or pH 5.0 acetate buffer) and transferred into a dialysis bag $(\mathrm{MWCO}=3500 \mathrm{Da})$. The bag was submerged into $15 \mathrm{~mL}$ of the same buffer and stirred at $37{ }^{\circ} \mathrm{C}(50 \mathrm{rpm}) .0 .3 \mathrm{~mL}$ aliquots were withdrawn from the dissolution medium at predetermined times and analysed with an Agilent Cary 100 spectrophotometer. To maintain a constant volume, $0.3 \mathrm{~mL}$ of fresh pre-heated buffer was added to the dissolution vessel at each time point. MTX and 5FU quantifications were performed at $\lambda_{\max }$ of $303 \mathrm{~nm}$ for MTX and $262 \mathrm{~nm}$ for 5FU. Dilutions were undertaken when necessary to bring concentrations into the linear range of the calibration curve. Experiments were performed in triplicate and the results are reported as mean \pm standard deviation (S.D.).

\section{Proton Relaxivity Measurements}

A MQC + benchtop NMR analyzer (Oxford Instruments) was used to measure longitudinal $\left(T_{1}\right)$ and transverse relaxation times $\left(T_{2}\right)$ of protons at $37{ }^{\circ} \mathrm{C}$ and $23 \mathrm{MHz}$. The standard inversion-recovery method was employed to determine $T_{1}$, with a typical $90^{\circ}$ pulse calibration of $250 \mu \mathrm{s}$ and 4 scans per experiment. The Carr-Purcell-MeiboomGill (CPMG) method was used to measure $T_{2}$, with 4 scans per experiment. The water relaxation rate enhancement per mmol of contrast agent is defined by Eq. 1 .

$r_{1,2}=\frac{R_{1,2, o b s}-R_{1,2, s o l}}{[C A]}$

where $R_{1,2, o b s}$ is the observed relaxation rate of the agent in aqueous suspension $\left(R_{l, 2}=1 / T_{1,2}\right), R_{l, 2, s o l}$ is the relaxation rate of the unaltered solvent system (i.e. in the absence of contrast agent) and $[\mathrm{CA}]$ is the $\mathrm{mM}$ concentration of the contrast agent in suspension, as measured by ICP-OES.

To monitor changes in proton relaxivity with $\mathrm{pH}$, a dispersion of approximately $16 \mathrm{mg}$ of each LDH sample in $8 \mathrm{~mL}$ of a $0.1 \%(\mathrm{w} / \mathrm{v})$ aqueous xanthan gum solution was placed into a $10 \mathrm{~mm}$-diameter NMR tube, which was held at $37{ }^{\circ} \mathrm{C}$. The longitudinal and transverse relaxation time $\left(T_{1}\right.$ and $T_{2}$ ) were directly monitored over $7 \mathrm{~h}$. At predetermined time points, $0.3 \mathrm{~mL}$ of suspension was taken from the NMR tube, diluted, and filtered through a PVDF-type syringe filter $(0.22 \mu \mathrm{m})$. Then centrifugation $(13,200 \mathrm{rpm}$ for $10 \mathrm{~min}$ ) was conducted to remove the remaining particles, and the drug concentration and free [Gd] were determined using UV-Vis spectroscopy and ICP-OES, respectively. All experiments were performed in triplicate and the results are reported as mean \pm S.D.

\section{Results}

\section{Preparation and Characterization of Drug Loaded Gd-LDHs}

The powder XRD patterns of the LDH materials are given in the Supporting Information, Fig. S1A. The XRD pattern of blank MgAl-LDH confirms the presence of a well crystalized hydrotalcite phase (JCPDS card 35-0965) and the visible peaks can be indexed to the LDH (003), (006), (009), (015), (018), (110), and (113) reflections. For Gd$\mathrm{LDH}$, the characteristic reflections of the LDH phase can again be observed, but the crystallinity of Gd-LDH is lower than that of the parent LDH. The basal reflections appear to comprise two overlapping peaks, indicating two slightly different interlayer spacings are present. The low crystallinity of the material also seems to cause the overlap of the (110) and (113) reflections in Gd-LDH, (see Fig. S1A). The presence of Gd leads to broader peaks that partially shift to higher angle, indicative of local structural difference induced by the relatively large Gd ion. To verify the presence of gadolinium in the nanosheets, X-ray photoelectron spectroscopy (XPS) was carried out, and the detailed Gd $4 p$ spectra (Fig. S1B) revealed a strong peak around $143 \mathrm{eV}$ and small peak at $148 \mathrm{eV}$. These can be assigned to $4 \mathrm{~d}_{5 / 2}$ and $4 \mathrm{~d}_{3 / 2}$ of $\mathrm{Gd}^{3+}$, respectively, and are typical of an octahedral coordination environment such as that found in an LDH [26]. According to elmental analysis (Table S1), the $[\mathrm{Mg}]:[\mathrm{Al}]:[\mathrm{Gd}]$ molar ratio of $\mathrm{Gd}-\mathrm{LDH}$ is close to the theoretical ratio $(2.1: 1: 0.05)$.

To obtain theranostics for MRI-guided chemotherapy, two chemotherapeutics, 5FU and MTX were loaded into pre-formed Gd-LDH via ion-exchange. Compared to the XRD patterns of blank Gd-LDH, the (003) and (006) reflections (Fig. 2) are broader and lower intensity after MTX or 5FU intercalation, as a result of the introduction of stacking defects $[27,28]$. The $(003)$ peak is also shifted 


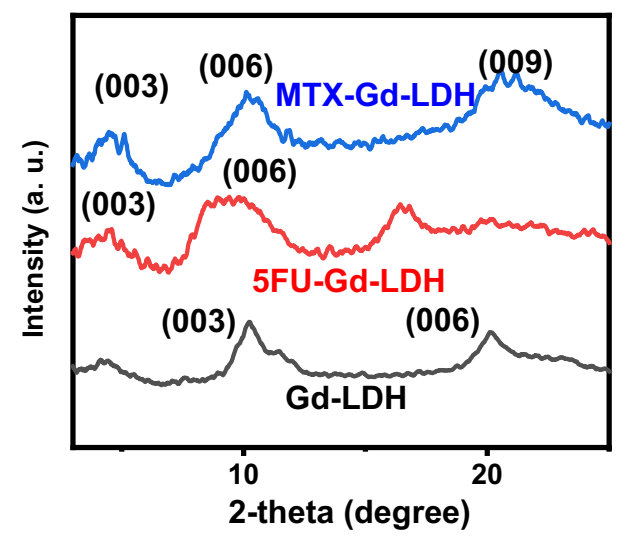

Fig. 2 XRD patterns of Gd-LDH, and Gd-LDH loaded with 5FU or MTX

towards lower angle because of an increase in the interlayer spacing to accommodate the larger drug ions [27, 28]. No reflections of the active pharmaceutical ingredients (APIs) (Fig. S1C) are observed in the XRD data, suggesting no pure drug crystals are present in the drug loaded LDHs. $[\mathrm{Mg}]:[\mathrm{Al}]:[\mathrm{Gd}]$ molar ratio remains similar to the pristine Gd-LDH as given in Table S1.

In TEM, Gd-LDH can be seen to exist as pseudohexagonal nanosheets (Fig. 3A). After drug loading, the LDHs manifest as aggregated plate-like nanoparticles (Fig. 3B, C). This is consistent with the literature [29]. The hydrodynamic diameter of Gd-LDH is $186 \pm 1 \mathrm{~nm}$ (PDI $0.29 \pm 0.00$ ), while 5FU-Gd-LDH was larger at $303 \pm 4 \mathrm{~nm}$ (PDI $0.28 \pm 0.01$ ), and MTX-LDH-Gd had a still greater particle size $(416 \pm 4 \mathrm{~nm}$, PDI $0.64 \pm 0.01)$. No endothermic melting peaks can be found in DSC profiles (Fig. 4A), confirming that there are no MTX crystals present in the reaction product (no conclusions can be drawn for $5 \mathrm{FU}$ here, since the intercalates begin to degrade before the melting point of the pure drug). FTIR spectra are shown in Fig. 4B. The stretching bands of both hydroxide and interlayer water molecules are found at approximately $3400 \mathrm{~cm}^{-1}$, and a band at $1647-48 \mathrm{~cm}^{-1}$ can be ascribed to the $\delta$-bend of water. The low intensity peak at around $1450 \mathrm{~cm}^{-1}$ in MTX-Gd-LDH can be attributed to stretching vibrations of $\mathrm{C}=\mathrm{C}$ bonds in MTX, and the peak at
$1610 \mathrm{~cm}^{-1}$ corresponds to the water bending mode. Further, a pair of absorption bands at 1387 and $1558 \mathrm{~cm}^{-1}$ arise from $\mathrm{COO}^{-}$stretching vibrations of the carboxylic acid groups of MTX. Similarly, successful intercalation of $5 \mathrm{FU}$ was confirmed by IR spectroscopy. There are a range of additional peaks characteristic of $5 \mathrm{FU}$ in 5FU-Gd-LDH. These arise around $1672 \mathrm{~cm}^{-1}(\mathrm{C}=\mathrm{O}$ and $\mathrm{C}=\mathrm{C}$ stretches), 1586-1300 and $809 \mathrm{~cm}^{-1}$ (ring stretching modes), and $1216 \mathrm{~cm}^{-1}$ (C-F vibrations) [30].

\section{Stability Test}

Free $\mathrm{Gd}^{3+}$ is toxic to humans [31]. Thus the stability of the nanocomposites was explored at $\mathrm{pH} 5.0$ (late endosome/ lysosome in cells), $\mathrm{pH} 6.5$ (tumour microenvironment) and pH 7.4 (representing the general physiological environment). Experiments were performed for $24 \mathrm{~h}$, and the [Gd] freed into the release medium was analysed using ICPOES. The results are given in Fig. 5. At pH 7.4, no Gd was detected, indicating the drug loaded Gd-LDHs are stable in neutral environments. At $\mathrm{pH} 6.5$ and 5.0, a small proportion of [Gd] was released after $24 \mathrm{~h}$, equating to a maximum of 2.4 (pH 6.5) and 2.9\% (pH 5.0) of the Gd content leaching for MTX-Gd-LDH, and $1.9(\mathrm{pH} 6.5)$ and $2.4 \%$ (pH 5.0) for 5FU-Gd-LDH. Hence, the Gd-LDH nanosheets are stable in most physiological environments $(\mathrm{pH}>$ 7.0), but free $\mathrm{Gd}^{3+}$ ions might be freed in acidic environments (e.g. tumour environments, GI tract) in vivo.

\section{In Vitro Drug Release}

The drug loadings of MTX-Gd-LDH and 5FU-Gd-LDH were calculated to be $34.6 \pm 5.9$ and $13.2 \pm 6.2 \mathrm{w} / \mathrm{w} \%$ (mean $\pm \mathrm{SD}, \mathrm{n}=5$ ), with encapsulation efficiency (EE) of $30.4 \pm 5.2$ and $13.5 \pm 6.4 \%$ respectively. The MTX loaded nanoparticles have a markedly higher drug loading capacity, which is consistent with the literature [32, 33]. This might arise due to the anionic carboxylate groups of MTX, which can be easily intercalated into the LDHs via ion exchange. In contrast, $5 \mathrm{FU}$ is amphoteric and bears a reduced net charge under the conditions used for intercalation. It is also more water-soluble, meaning that
Fig. 3 TEM images of A GdLDH, B 5FU-Gd-LDH (B) and C MTX-Gd-LDH
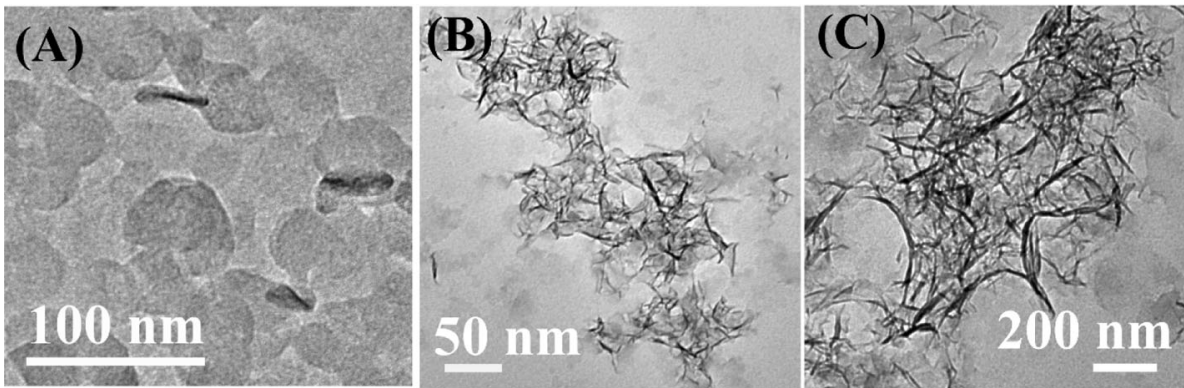

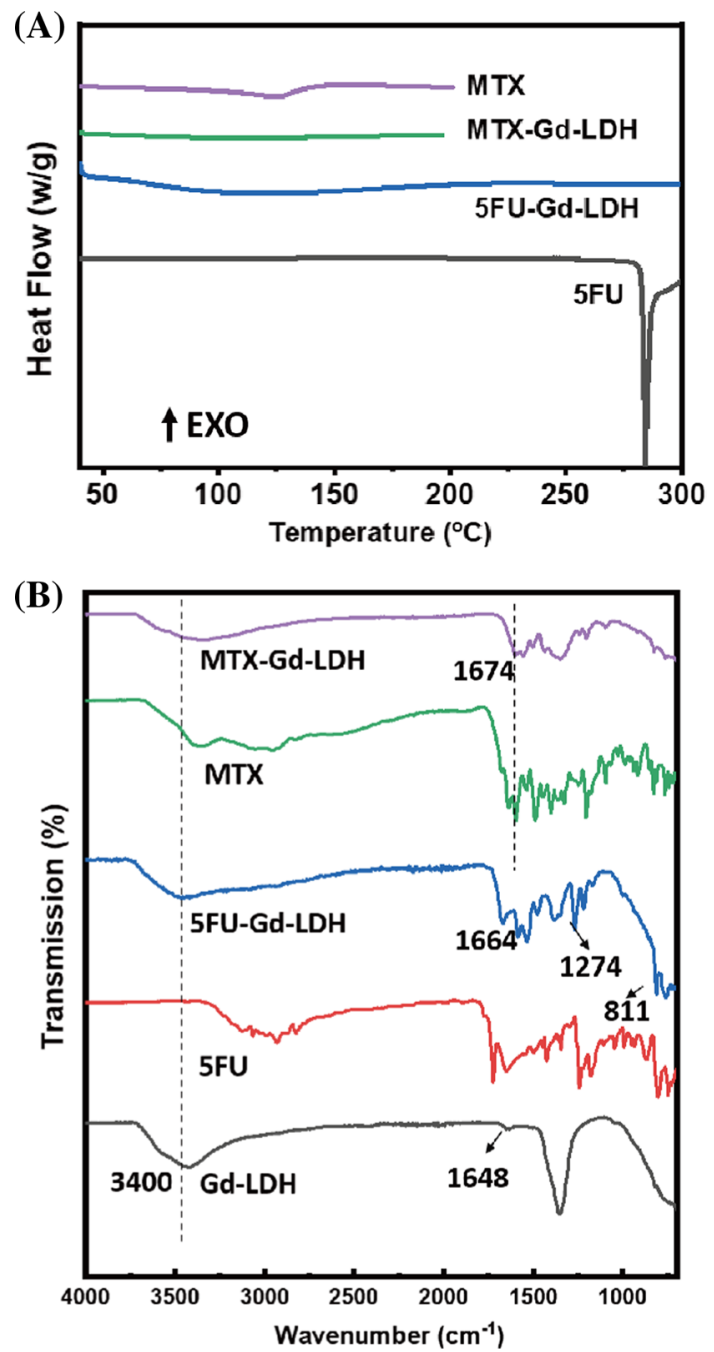

Fig. 4 A DSC profile of the drug loaded Gd-LDH samples and raw materials; B FTIR spectra of MTX-Gd-LDH, 5FU-Gd-LDH, pristine Gd-LDH, MTX and 5FU thermodynamically it is more favourable for the $5 \mathrm{FU}$ ions to remain in solution.

Drug release was explored at $\mathrm{pH} 5.0,6.5$ and 7.4. The results are presented in Fig. 6. A burst release of drug from the $\mathrm{LDH}$ can be found in all cases. The LDHs exhibit $\mathrm{pH}-$ responsive release rates. For MTX-Gd-LDH, the final release percentage $(48 \mathrm{~h})$ is around $80 \%$ at $\mathrm{pH} 5.0,67 \%$ at $\mathrm{pH} 6.5$, and $57 \%$ at $\mathrm{pH} 7.4$. The release of 5FU-Gd-LDH reached the highest release percentage after around $7 \mathrm{~h}$ (approx. $88 \%$ at pH, $75 \%$ at $\mathrm{pH} 6.5$ and $70 \%$ at $\mathrm{pH} 7.4$ ), followed by a slight decline to ca. $86 \%(\mathrm{pH} 5.0), 73 \%(\mathrm{pH}$ 6.5) and 67\% (pH 7.4) after $48 \mathrm{~h}$, likely to be due to some drug crystallising out of solution or adsorbing back onto the LDH composite [27].

The Bhaskar model was applied to analyse the drug release data. This assumes that diffusion through a particle is the rate limiting step to release, making it appropriate for ion-exchange processes [34]. $M_{t}$ is the amount of drug released at time $t, M_{i n f}$ the total amount of drug loaded in the carrier, and $k$ is a rate constant.

$\ln \left(1-\frac{M_{t}}{M_{\text {inf }}}\right)=k t^{0.65}$

Fits of the Bhaskar model to the release data are shown in Fig. S2. The release data in general can be well fitted to this model in all three $\mathrm{pH}$ environments. The Bhaskar equation assumes that the that drug release rate is dependent on the diffusion of the drug ions and/or replacement anions through the system. Thus, most drug release $(\sim 70 \%)$ can be regarded to be an ion-exchange controlled process, which occurred in the first $2 \mathrm{~h}$.

The Avarmi-Erofe'ev model (Eq. 3) [35] can be used to study how solids transform from one phase to another at constant temperature. Here, $\alpha=M_{t} / M_{i n f}, n$ is an exponent that provides information on the reaction mechanism, and $k$ is a rate constant.
Fig. $5[\mathrm{Gd}]$ released from A MTX-Gd-LDH and B 5FUGd-LDH at pH 6.5 and 5.0. Data are given from three independent experiments as mean \pm S.D
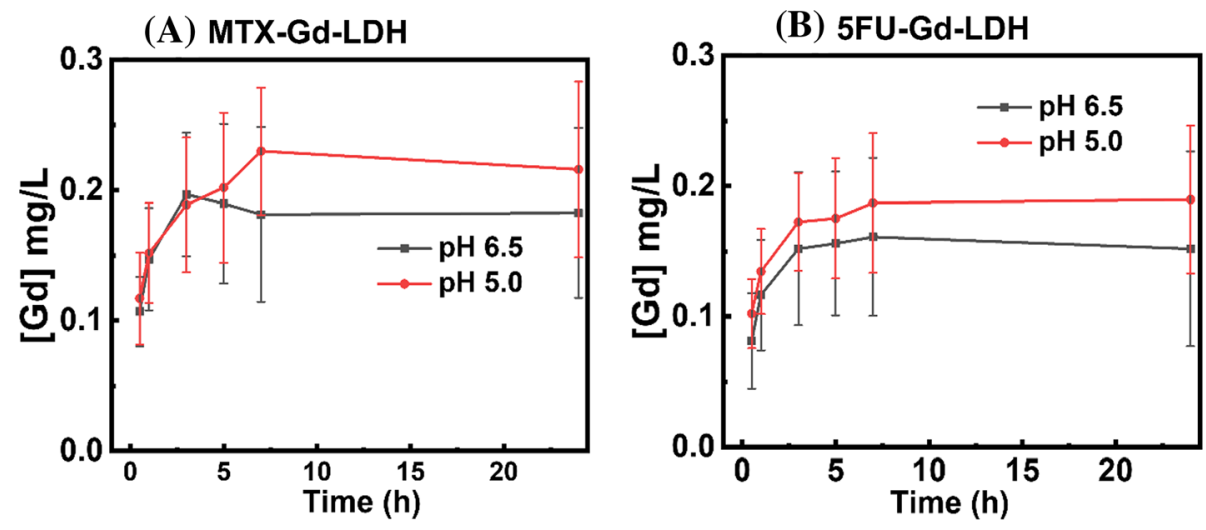
Fig. 6 Drug release profiles of A 5FU-Gd-LDH and B MTXGd-LDH. Data are given from three independent experiments as mean \pm S.D
(A)

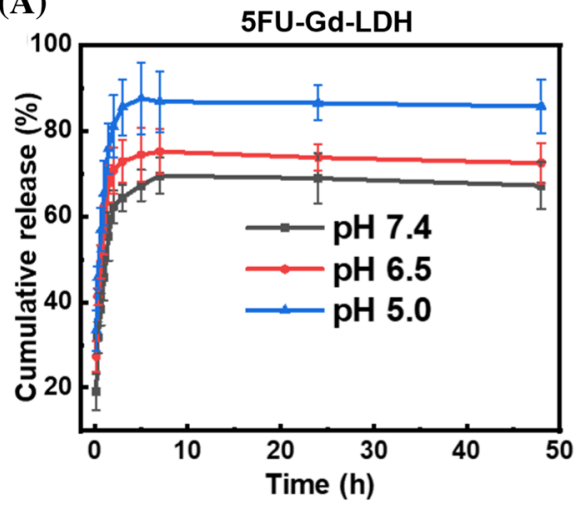

(B)

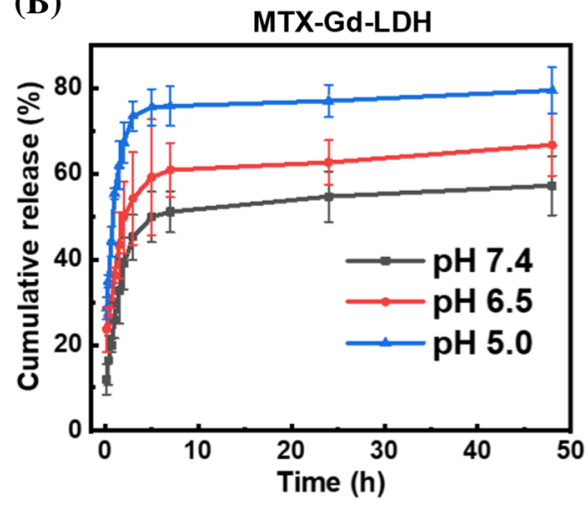

Table 1 The values of the $n$ exponent and $\mathrm{R}^{2}$ determined from the Avarmi-Erofe'ev model, using the data obtained from the first $2 \mathrm{~h}$ $(\sim 60 \%)$ of drug release

\begin{tabular}{llll}
\hline & $\mathrm{pH}$ & $n$ & $\mathrm{R}^{2}$ \\
\hline 5FU-Gd-LDH & 7.4 & 0.58 & 0.9810 \\
& 6.5 & 0.58 & 0.9841 \\
& 5.0 & 0.56 & 0.9952 \\
MTX-Gd-LDH & 7.4 & 0.60 & 0.9700 \\
& 6.5 & 0.37 & 0.9090 \\
& 5.0 & 0.50 & 0.9837 \\
\hline
\end{tabular}

Table 2 The longitudinal $\left(r_{1}\right)$ and transverse $\left(r_{2}\right)$ relaxivity of pristine Gd-LDH (pH 7.0)

\begin{tabular}{llcl}
\hline Sample & $r_{I}\left(\mathrm{mM}^{-1} \mathrm{~s}^{-1}\right)$ & $r_{2}\left(\mathrm{mM}^{-1} \mathrm{~s}^{-1}\right)$ & $r_{2} / r_{I}$ \\
\hline Gd-LDH & $9.5 \pm 1.2$ & $11.0 \pm 1.8$ & 1.16 \\
Gd(DTPA) & $4.5 \pm 0.6$ & $5.0 \pm 0.8$ & 1.12 \\
\hline
\end{tabular}

$\ln (-\ln (1-a))=n \ln k+n \ln t$

As shown in Fig. S3 and Table 1, the Avarmi-Erofe'ev model also fits the release data. The value of $n$ provides some information on the reaction mechanism. The values of $n$ lie in the range of 0.37 to 0.60 . All of these suggest diffusion control, indicating that diffusion of replacement ions through the solution/interlayer space limits the release rate. This is consistent with the fitting results obtained with the Bhaskar model. However, it should be noted that there are a range of potential interpretations of the $n$ values obtained, and thus it is not possible to draw definitive conclusions here.

\section{Relaxivity}

Firstly, the longitudinal and transverse relaxivity of pristine Gd-LDH and a clinically used Gd-based contrast agent, Gd(DTPA) were recorded (see Table 2). Clearly, the GdLDH shows higher relaxivity than Gd(DTPA), and can potentially be used as a $T_{1}$ contrast agent.

For drug loaded Gd-LDHs, we investigated the relaxation behaviour of 5FU-Gd-LDH and MTX-Gd-LDH at different $\mathrm{pHs}$ over $24 \mathrm{~h}$, since the release of the drug might affect the relaxation behavior of the paramagnetic centres. The free $[\mathrm{Gd}]$ was also monitored to elucidate any relationship between $[\mathrm{Gd}]$ and proton relaxivity. As shown in Fig. 7, the $r_{1}$ values of MTX-Gd-LDH remained low and roughly unchanged at neutral $\mathrm{pH}\left(\sim 0.83 \mathrm{mM} \mathrm{s}^{-1}\right)$, while in acidic environments the $r_{l}$ values are higher, increasing to $2.3 \mathrm{mM} \mathrm{s}^{-1}$ at $\mathrm{pH} 6.5$ and $2.7 \mathrm{mM} \cdot \mathrm{s}^{-1}$ at $\mathrm{pH} 5.0$ after $24 \mathrm{~h}$. The $r_{1}$ profiles of 5FU-Gd-LDH showed similar trends (Fig. 7).

The corresponding drug and [Gd] freed into the xanthan gum solution used for relaxivity experiments are given in Fig. 7C-F. Both drug loaded Gd-LDHs displayed higher relaxivity $\left(r_{1}\right)$ in acidic $\mathrm{pH}$, since a small proportion of $\mathrm{Gd}$ was released into the acidic buffer, thus promoting interactions between $\mathrm{Gd}^{3+}$ paramagnetic centres and water protons. The $\mathrm{pH}$-sensitive relaxation behaviour potentially could allow the particles to be used for reporting on the acidic tumour microenvironment in the human body.

The drug loaded Gd-LDH nanosheets displayed rapid release profiles, reaching a plateau after $\sim 3 \mathrm{~h}$, consistent with previous reports in the literature [27, 36] and the separate drug release experiments discussed above (Fig. 6). The release is also $\mathrm{pH}$-responsive, and more drug was freed in acidic conditions as a result of the LDH layers weathering in the presence of $\mathrm{H}^{+}$[37]. Compared to pristine $\mathrm{Gd}$ - 
Fig. 7 The longitudinal relaxivity $\left(r_{l}\right)$ profile of $\mathbf{A} 5 \mathrm{FU}$ Gd-LDH and B MTX-Gd-LDH, with drug release profiles of C 5FU-Gd-LDH and D MTXGd-LDH, and [Gd] release from E 5FU-Gd-LDH and F MTXGd-LDH
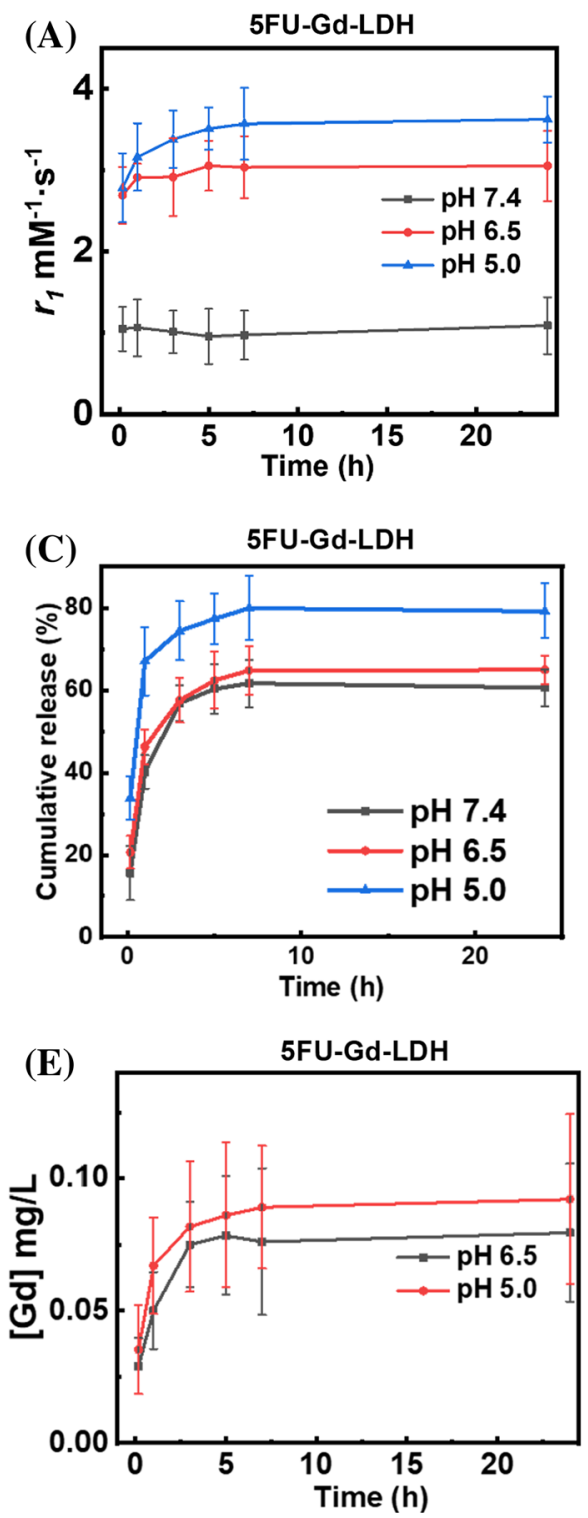
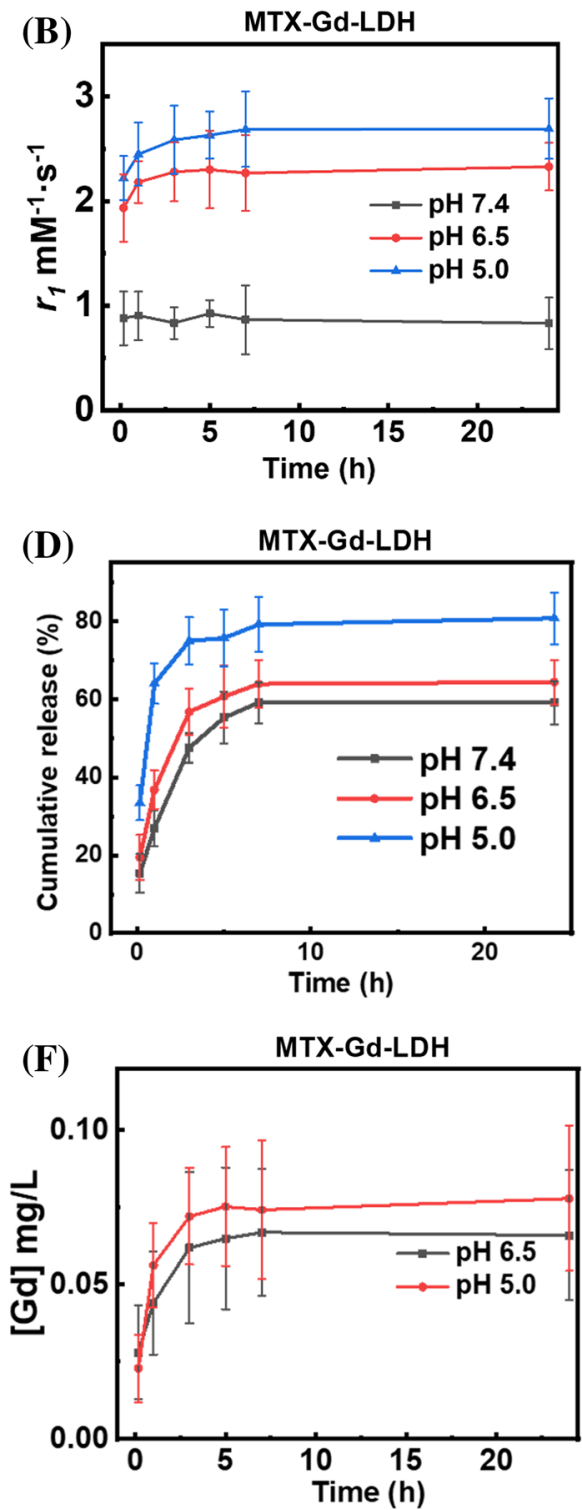

LDH, the longitudinal relaxivity $\left(r_{l}\right)$ values decreased after drug loading. This might be because the drug ions intercalated are more hydrophobic than the original guest anions $\left(\mathrm{Cl}^{-}\right)$, weakening the corporation between the paramagnetic centres $\left(\mathrm{Gd}^{3+}\right)$ and water protons. However, the $r_{1}$ values $\left(\sim 1-4 \mathrm{mM} \mathrm{s}^{-1}\right)$ are still relatively close to Gd(DTPA) $\left(4.5 \mathrm{mM} \mathrm{s}^{-1}\right)$, indicating acceptable $T_{1}$ MRI contrast ability.

\section{Conclusion}

In this work, a $\mathrm{Gd}^{3+}$ doped LDH obtained by a 'bottom-up' method was used to intercalate two cytotoxic agents, 5FU and MTX, via ion-exchange. X-ray diffraction patterns displayed expanded interlayer spacings confirming successful intercalation of the drug ions. IR spectra revealed the presence of characteristic peaks of MTX or $5 \mathrm{FU}$ in the intercalates. A stability assessment found that the Gd-doped LDHs were stable under physiological conditions, while in acidic conditions a small proportion of $\mathrm{Gd}$ was freed into the immersion medium. During in vitro release studies both 5FU and MTX were rapidly released from the $\mathrm{LDH}$ carrier, in a manner responsive to acidic $\mathrm{pH}$. The longitudinal relaxivity of the Gd-LDHs remains roughly stable during drug release over $24 \mathrm{~h}$, and was higher in acidic conditions. Hence, the drug-loaded GdLDH nansheets produced can potentially serve as $\mathrm{pH}-$ sensitive theranostic platforms for MRI-guided chemotherapy. 
Supplementary Information The online version contains supplementary material available at https://doi.org/10.1007/s10876022-02226-5.

Acknowledgements The authors gratefully thank Dr Andrew Weston for assistance with electron microscopy experiments.

\section{Declarations}

Conflict of interest GRW is a co-Editor-in-Chief of the Journal of Cluster Science.

Open Access This article is licensed under a Creative Commons Attribution 4.0 International License, which permits use, sharing, adaptation, distribution and reproduction in any medium or format, as long as you give appropriate credit to the original author(s) and the source, provide a link to the Creative Commons licence, and indicate if changes were made. The images or other third party material in this article are included in the article's Creative Commons licence, unless indicated otherwise in a credit line to the material. If material is not included in the article's Creative Commons licence and your intended use is not permitted by statutory regulation or exceeds the permitted use, you will need to obtain permission directly from the copyright holder. To view a copy of this licence, visit http://creativecommons. org/licenses/by/4.0/.

\section{References}

1. Z. Cao, B. Li, L. Sun, L. Li, Z. P. Xu, and Z. Gu (2020). 2D layered double hydroxide nanoparticles: recent progress toward preclinical/clinical nanomedicine. Small Methods 4, 1900343.

2. G. Simoneau (1996). Absence of rebound effect with calcium carbonate. Eur. J. Drug Metab. Pharmacokinet. 21, 351-357.

3. B. Saifullah, M. Z. Hussein, S. H. Hussein-Al-Ali, P. Arulselvan, and S. Fakurazi (2013). Antituberculosis nanodelivery system with controlled-release properties based on para amino salicylate-zinc aluminum-layered double-hydroxide nanocomposites. Drug Des. Dev. Ther. 7, 1365.

4. G. Mishra, B. Dash, and S. Pandey (2018). Layered double hydroxides: A brief review from fundamentals to application as evolving biomaterials. Appl. Clay Sci. 153, 172-186.

5. K. Sugano, M. Kansy, P. Artursson, A. Avdeef, S. Bendels, L. Di, G. F. Ecker, B. Faller, H. Fischer, G. Gerebtzoff, H. Lennernaes, and F. Senner (2010). Coexistence of passive and carrier-mediated processes in drug transport. Nat. Rev. Drug Discov. 9, 597-614.

6. M. Z. Hussein, S. H. Al Ali, Z. Zainal, and M. N. Hakim (2011). Development of antiproliferative nanohybrid compound with controlled release property using ellagic acid as the active agent. Int. J. Nanomed. 6, 1373.

7. V. Rives, M. Del Arco, and C. Martín (2013). Layered double hydroxides as drug carriers and for controlled release of nonsteroidal antiinflammatory drugs (NSAIDs): a review. J. Controll. Release 169, 28-39.

8. V. Rives, M. Del Arco, and C. Martín (2014). Intercalation of drugs in layered double hydroxides and their controlled release: a review. Appl. Clay Sci. 88, 239-269.

9. Z. Wang, E. Wang, L. Gao, and L. Xu (2005). Synthesis and properties of $\mathrm{Mg}_{2} \mathrm{Al}$ layered double hydroxides containing 5-fluorouracil. J. Solid State Chem. 178, 736-741.

10. G. L. Davies, I. Kramberger, and J. J. Davis (2013). Environmentally responsive MRI contrast agents. Chem. Commun. 49, 9704-9721.
11. M. D. Rowe, D. H. Thamm, S. L. Kraft, and S. G. Boyes (2009). Polymer-modified gadolinium metal-organic framework nanoparticles used as multifunctional nanomedicines for the targeted imaging and treatment of cancer. Biomacromolecules 10, 983-993.

12. W. Hatakeyama, T. J. Sanchez, M. D. Rowe, N. J. Serkova, M. W. Liberatore, and S. G. Boyes (2011). Synthesis of gadolinium nanoscale metal-organic framework with hydrotropes: manipulation of particle size and magnetic resonance imaging capability. ACS Appl. Mater. Interfaces 3, 1502-1510.

13. J. S. Ananta, B. Godin, R. Sethi, L. Moriggi, X. Liu, R. E. Serda, R. Krishnamurthy, R. Muthupillai, R. D. Bolskar, L. Helm, M. Ferrari, L. J. Wilson, and P. Decuzzi (2010). Geometrical confinement of gadolinium-based contrast agents in nanoporous particles enhances T1 contrast. Nat. Nanotechnol. 5, 815-821.

14. E. N. M. Cheung, R. D. Alvares, W. Oakden, R. Chaudhary, M. L. Hill, J. Pichaandi, and R. S. Prosser (2010). Polymer-stabilized lanthanide fluoride nanoparticle aggregates as contrast agents for magnetic resonance imaging and computed tomography. Chem. Mater. 22, 4728-4739.

15. O. Icten, D. A. Kose, S. J. Matissek, J. A. Misurelli, S. F. Elsawa, N. S. Hosmane, and B. Zumreoglu-Karan (2018). Gadolinium borate and iron oxide bioconjugates: nanocomposites of next generation with multifunctional applications. Mater. Sci. Eng. C 92, 317-328.

16. F. Söderlind, H. Pedersen, R. M. Petoral, P. Käll, and K. Uvdal (2005). Synthesis and characterisation of $\mathrm{Gd}_{2} \mathrm{O}_{3}$ nanocrystals functionalised by organic acids. J. Colloid Interface Sci. 288, 140-148.

17. Y. Hou, R. Qiao, F. Fang, X. Wang, C. Dong, K. Liu, C. Liu, Z. Liu, H. Lei, F. Wang, and M. Gao (2013). $\mathrm{NaGdF}_{4}$ nanoparticlebased molecular probes for magnetic resonance imaging of intraperitoneal tumor xenografts in vivo. Acs Nano 7, 330-338.

18. Y. Xu, A. Goyanes, Y. Wang, A. J. Weston, P. W. So, C. F. Geraldes, A. M. Fogg, A. W. Basit, and G. R. Williams (2018). Layered gadolinium hydroxides for simultaneous drug delivery and imaging. Dalton Trans. 47, 3166-3177.

19. H. Zuo, W. Chen, B. Li, K. Xu, H. Cooper, Z. Gu, and Z. P. Xu (2017). MnAl layered double hydroxide nanoparticles as a dualfunctional platform for magnetic resonance Imaging and siRNA delivery. Chem. Eur. J. 23, 14299.

20. B. Li, J. Tang, W. Chen, G. Hao, N. Kurniawan, Z. Gu, and Z. P. Xu (2018). Novel theranostic nanoplatform for complete mice tumor elimination via MR imaging-guided acid-enhanced photothermo-/chemo-therapy. Biomaterials 177, 40-51.

21. W. Xie, Z. Guo, Z. Cao, Q. Gao, D. Wang, C. Boyer, M. Kavallaris, X. Sun, X. Wang, L. Zhao, and Z. Gu (2019). Manganese-based magnetic layered double hydroxide nanoparticle: a $\mathrm{pH}$-sensitive and concurrently enhanced $\mathrm{T} 1 / \mathrm{T} 2$-weighted dualmode magnetic resonance imaging contrast agent. ACS Biomater. Sci. Eng. 5, 2555-2562.

22. T. Jia, Z. Wang, Q. Sun, S. Dong, J. Xu, F. Zhang, L. Feng, F. He, D. Yang, P. Yang, and J. Lin (2020). Intelligent Fe-Mn layered double hydroxides nanosheets anchored with upconversion nanoparticles for oxygen-elevated synergetic therapy and bioimaging. Small 16, 2001343.

23. Z. Wang, L. Fu, Y. Zhu, S. Wang, G. Shen, L. Jin, and R. Liang (2021). Chemodynamic/photothermal synergistic therapy based on $\mathrm{Ce}$-doped $\mathrm{Cu}-\mathrm{Al}$ layered double hydroxides. J. Mater. Chem. B 9, 710-718.

24. M. S. Usman, M. Z. Hussein, S. Fakurazi, and F. F. A. Saad (2017). Gadolinium-based layered double hydroxide and graphene oxide nano-carriers for magnetic resonance imaging and drug delivery. Chem. Central J. 11, 1-10.

25. B. Li, Z. Gu, N. Kurniawan, W. Chen, and Z. P. Xu (2017). Manganese-based layered double hydroxide nanoparticles as a 
T1-MRI contrast agent with ultrasensitive $\mathrm{pH}$ response and high relaxivity. Adv. Mater. 29, 1700373.

26. X. Y. Ren, X. X. Yuan, Y. P. Wang, C. L. Liu, Y. Qin, L. P. Guo, and L. H. Liu (2016). Facile preparation of $\mathrm{Gd}^{3+}$ doped carbon quantum dots: photoluminescence materials with magnetic resonance response as magnetic resonance/fluorescence bimodal probes. Opt. Mater. 57, 56-62.

27. Y. Wang, Z. Zhang, Y. Abo-zeid, J. C. Bear, G. L. Davies, X. Lei, and G. R. Williams (2020). $\mathrm{SiO}_{2}$-coated layered gadolinium hydroxides for simultaneous drug delivery and magnetic resonance imaging. J. Solid State Chem. 286, 121291.

28. L. Li, Z. Gu, W. Gu, J. Liu, and Z. P. Xu (2016). Efficient drug delivery using $\mathrm{SiO}_{2}$-layered double hydroxide nanocomposites. $J$. Colloid Interface Sci. 470, 47-55.

29. M. Chakraborty, S. Dasgupta, P. Bose, A. Misra, T. K. Mandal, M. Mitra, J. Chakrabortya, and D. Basu (2011). Layered double hydroxide: inorganic organic conjugate nanocarrier for methotrexate. J. Phys. Chem. Solids 72, 779-783.

30. G. Tuncelli, A. N. Ay, and B. Zümreoglu-Karan (2015). 5-Fluorouracil intercalated iron oxide@ layered double hydroxide core-shell nano-composites with isotropic and anisotropic architectures for shape-selective drug delivery applications. Mater. Sci. Eng. C 55, 562-568.

31. M. Rogosnitzky and S. Branch (2016). Gadolinium-based contrast agent toxicity: a review of known and proposed mechanisms. Biometals 29, 365-376.
32. M. Chakraborty, S. Dasgupta, S. Sengupta, J. Chakraborty, S. Ghosh, J. Ghosh, M. K. Mitra, A. Mishra, T. K. Mandal, and D. Basu (2012). A facile synthetic strategy for $\mathrm{Mg}-\mathrm{Al}$ layered double hydroxide material as nanocarrier for methotrexate. Ceram. Int. 38, 941-949.

33. Q. J. Huang, H. Y. Zeng, W. Zhang, B. Feng, X. J. Liu, H. Z. Duan, and P. X. Ding (2016). Loading kinetics of 5-fluorouracil onto hydrotalcite and in vitro drug delivery. J. Taiwan Inst. Chem. Eng. 60, 525-531.

34. R. Bhaskar, R. S. R. Murthy, B. D. Miglani, and K. Viswanathan (1986). Novel method to evaluate diffusion controlled release of drug from resinate. Int. J. Pharm. 28, 59-66.

35. S. Hulbert (1969). Models for solid-state reactions in powdered com pacts. J. Br. Ceram. Soc. 6, 11-20.

36. L. Jin, Q. Liu, Z. Sun, X. Ni, and M. Wei (2010). Preparation of 5 -fluorouracil/ $\beta$-cyclodextrin complex intercalated in layered double hydroxide and the controlled drug release properties. Ind. Eng. Chem. Res. 49, 11176-11181.

37. M. L. Parello, R. Rojas, and C. E. Giacomelli (2010). Dissolution kinetics and mechanism of $\mathrm{Mg}-\mathrm{Al}$ layered double hydroxides: a simple approach to describe drug release in acid media. J. Colloid Interface Sci. 351, 134-139.

Publisher's Note Springer Nature remains neutral with regard to jurisdictional claims in published maps and institutional affiliations. 